\title{
Influencia del empaque y envasado sobre las propiedades fisicoquímicas del hongo comestible Pleurotus ostreatus
}

\section{Influences of the packing and packed about physiochemical properties of edible mushroom Pleurotus ostreatus.}

\author{
Misael Cortés R, ${ }^{* 1}$ Ph.D, Marilza Ruiz R, ${ }^{1}$ M.Sc, Luís Henriquez,${ }^{1}$ Ing. Agrícola.
}

\begin{abstract}
${ }^{1}$ Universidad Nacional de Colombia Sede Medellín, Facultad de Ciencias Agropecuarias, Departamento de Ingeniería Agrícola y de Alimentos, Calle 59a No. 63 - 20, Medellín, Colombia. *Correspondencia: mcortesro@unal.edu.co
\end{abstract}

Recibido: Agosto de 2009; Aceptado: Diciembre de 2010.

\section{RESUMEN}

Objetivo. Evaluar la influencia de diferentes empaques y atmósferas sobre las propiedades fisicoquímicas del hongo comestible Pleurotus ostreatus. Materiales y métodos. Los hongos fueron almacenados durante 15 días a $4^{\circ} \mathrm{C}$, utilizando tres empaques: 1) espuma de poliestireno con película de recubrimiento de polivinil cloruro (empaque comercial), 2) Polietilentereftalato con películas de recubrimiento de polipropileno biorientado y 3) polietileno de baja densidad y tres atmósferas de envasado: 1) Aire, 2) $100 \% \mathrm{~N}_{2}$ y 3) $10 \% \mathrm{O}_{2}, 10 \% \mathrm{CO}_{2}$ y $80 \% \mathrm{~N}_{2}$. Después de almacenados se derminaron los posibles cambios de las variables fisicoquímicas $\left(\mathrm{pH}\right.$, acidez, ${ }^{\circ}$ Brix, humedad, cloruros, color y textura) Resultados. Las muestras presentaron diferencias estadísticas en los parámetros fisicoquímicos del hongo fresco por efecto de los factores tiempo, empaque y atmósfera. Los rangos de variación se consideraron aceptables debido a las características propias del producto, como ente biológico que continua con sus procesos metabólicos. Los cambios de color en las condiciones de control (Grupo 1: Empacado Comercial y Grupo 1: Atmósfera Aire) no fueron muy acentuados, siendo instrumentalmente más apreciable, sin llegar a serlo para el observador. La textura en todos los casos presentó una disminución en la resistencia mecánica, debido a los posibles procesos fermentativos y al deterioro por la alta tasa metabólica. Conclusiones. Los resultados permiten identificar que no hubo un efecto apreciable del empaque y de las atmósferas modificadas en las propiedades fisicoquímicas del hongo Pleurotus ostreatus, lo que hace que el empaque comercial sea más práctico por efecto de costos de producción.

Palabras clave: Atmósfera, hongo, Pleurotus ostreatus, propiedades fisicoquímicas. (Fuente: AIMS). 


\section{ABSTRACT}

Objective. To determine the effect of different packings and modified atmospheres on the physiochemical properties of a comestible mushroom (Pleurotus ostreatus) in fresh state. Materials and methods. Mushrooms were stored at $4^{\circ} \mathrm{C}$ during 15 days, using three different forms of packings: 1) Polyestiren foam with polyvinyl chloride film cover (commercial packing), 2) Polyethylene terephthalate with polypropylene bioriented film cover and 3) Polyethylene of low density film cover and three different atmospheres: 1) Air, 2) $100 \% \mathrm{~N}_{2}$ and 3) $10 \% \mathrm{O}_{2}, 10 \% \mathrm{CO}_{2}$, and $80 \% \mathrm{~N}_{2}$. After stored possible changes in the physicochemical variables $\left(\mathrm{pH}\right.$, acidity, ${ }^{\circ}$ Brix, moisture, chlorides, color and texture were determined. Results. There was an effect of time, packing and atmosphere on the physiochemical properties of fresh mushrooms. The range of variations was considered acceptable due to the characteristics of the mushrooms, such as biological entity that continuous with their metabolic processes. The changes in color (Group1: commercial packing and Group 1: Air stored) were not very appreciable, for the observer in all groups. The texture presented a decrease in the mechanical resistance, these may be due to the possible fermentative processes and deterioration for the high metabolic rate. Conclusions. There was not an appreciable effect of packing and environmental atmospheric conditions on the physiochemical properties of the mushroom Pleurotus ostreatus. It was also found that the commercial packing is more practical by issues of production costs.

Key words: Atmosphere, Mushroom, Pleurotus ostreatus, physiochemical properties. (Source: AIMS).

\section{INTRODUCCIÓN}

El Pleurotus ostreatus es un hongo comestible, reconocido por su alto valor nutritivo. En los últimos años, debido al cambio en los hábitos alimenticios, se ha registrado un aumento creciente en su consumo per cápita, asociado a su bajo aporte calórico, una baja relación de ácidos grasos saturados a insaturados, 2.04.5:1, una relación fibra dietaría total a fibra cruda mayor que la de los vegetales, una buena digestibilidad (67.75 $\pm 0.54 \%)$ $(1,2)$, al contenido en compuestos funcionales (betaglucano y glucosamina), aminoácidos esenciales, minerales, vitaminas y provitaminas $(3,4)$. Además se le atribuyen propiedades medicinales (anticolesterolémica y antitumorales) y antioxidantes (5-7).

El consumo per cápita mundial de setas y hongos en el 2001 se estimó en $0.44 \mathrm{Kg}$ por persona al año, con un crecimiento promedio anual de $3.8 \%$. Este consumo es jalonado por países asiáticos, aunque en los últimos años, ha tendido a generalizarse a países occidentales, presentando un interesante potencial de mercado (8).
La calidad de los hongos es influenciada por diferentes parámetros, como el estado de desarrollo, las condiciones pre y poscosecha y el tipo de sustrato en el que son cultivados. El Pleurotus ostreatus es un hongo con un alto contenido de humedad, susceptible al ataque microbiológico, a las reacciones de pardeamiento enzimático (9) y al daño mecánico, debido a su estructura epidérmica delgada y porosa. La respiración de los hongos en general, es alta (200-500 $\mathrm{mg} / \mathrm{kg} \mathrm{h}$ a $20^{\circ} \mathrm{C}$ ), comparado con otras verduras y frutas (10), por lo que requieren mecanismos de empaque más selectivos que promuevan el mantenimiento de las características organolépticas y nutritivas. Para su comercialización, los hongos comestibles son empacados normalmente en bandeja de poliestireno (icopor), recubiertos con una película elástica de polietileno o cloruro de polivinilo (PVC), la cual es adherida al empaque y almacenados en refrigeración a $4^{\circ} \mathrm{C}(11)$.

Algunos autores han evaluado el efecto producido por el envasado en atmosfera modificada (EAM) en frutas y vegetales 
durante los últimos años con resultados positivos (12).

Algunas investigaciones realizadas en diferentes variedades de hongos comestibles EAM, han demostrado un efectivo control sobre su deterioro: Agáricus bisporus (10,13-16) Agrocybe chaxingu (17); Shiitake Lentinula edades (18). Estudios específicos, han establecido atmósferas modificas de 5-10 $\mathrm{kPa} \mathrm{O}_{2}+$ 2.5-5 kpa de $\mathrm{CO}_{2}$ en Agaricus bisporus (19), $5 \% \mathrm{O}_{2}, 3 \% \mathrm{CO}_{2}$ (15) $5 \% \mathrm{O}_{2}+10 \%$ $\mathrm{CO}_{2}(14)$ y de $1 \mathrm{kPa} \mathrm{O}+5 \mathrm{kPa} \mathrm{CO}{ }_{2} \mathrm{a}^{\circ} \mathrm{C}$ por 14 días (20), $1 \mathrm{kPa} \mathrm{O}_{2}, / 30 \mathrm{kPa} \mathrm{CO}_{2}$ (21), $12-15 \mathrm{kPa} \mathrm{O}+5 \mathrm{kPa} \mathrm{CO}_{2}$ (11) para Pleurotus sp.

Por otro lado, algunos autores han encontrado que el EAM puede tener un efecto negativo en algunas variedades de hongos, causado por la respiración anaeróbica como potencializador del crecimiento de microorganismos patógenos o por la acumulación excesiva de dióxido de carbono dentro del empaque, causando daños fisiológicos y severo pardeamiento $(11,14,17)$.

Investigaciones específicas, sobre el efecto en la vida útil del Pleurotus ostreatus, son escasas, aunque se ha confirmado que bajo este tratamiento se puede lograr una extensión de la vida útil, debido a la reducción del agua condensada, aunque sin llegar a eliminarse completamente (11).

El objetivo de este estudio fue evaluar la influencia de diferentes empaques y atmósferas modificadas sobre las propiedades fisicoquímicas del hongo comestible Pleurotus ostreatus en estado fresco.

\section{MATERIALES Y MÉTODOS}

Materia prima. Se utilizaron hongos enteros comestibles de la variedad Pleurotus ostreatus con un rango de peso de 3 a $12 \mathrm{~g}$, suministrados por la empresa Bioecológicos S.A (Rionegro, Antioquia), seleccionados por apariencia y calidad.
Caracterización fisicoquímica. Se determinó la acidez expresada en \% de ácido cítrico por $100 \mathrm{~g}$ de producto fresco, por titulación con $\mathrm{NaOH} 0.1 \mathrm{~N}$, según la norma técnica colombiana NTC 4623 (22); el \% cloruros por el método de Mohr NTC 210 (23); el \% de humedad (base húmeda: $H$ ) por la norma de la AOAC 7.003 y 930.15 adaptado (24); pH con potenciómetro (Hanna Instruments, Hanna 211, USA); sólidos solubles según NTC 4624 expresados como ${ }^{\circ}$ Brix a $20^{\circ} \mathrm{C}$ (Refractómetro Carlzeiss Jena, USA) (22); actividad de agua $\left(a_{w}\right)$ con un higrómetro de punto de rocío (Aqualab Decagón 3TE, USA). El color se determinó sobre las caras lisas $(C L)$ superficie superior del sombrero y rugosas (CR) laminillas en la parte inferior del sombrero, cada una como un factor para análisis estadístico, debido a las características únicas de reflexión de la luz de cada una de las caras, utilizando espectrocolorímetro (X-RITE SP64,BUSA) iluminante D65 y observador de $10^{\circ}$ como referencia; a partir de los espectros de reflexión se obtuvieron las coordenadas de color del CIE-L*a*b, donde $L^{*}$ es un indicador de la luminosidad, a* [cromaticidad verde (-) a rojo $(+)$ ] y b* [cromaticidad azul (-) a amarillo $(+)$ ]. La textura (dureza) del producto, se determinó en la $\mathrm{CL}$, a partir de ensayos de punción utilizando un analizador de textura (TA.XT2i Stable Micro Systems, USA), embolo metálico de $2 \mathrm{~mm}$ de diámetro y una velocidad de penetración de $4 \mathrm{~mm} / \mathrm{s}$ hasta una distancia $5 \mathrm{~mm}$.

Almacenamiento. Las muestras de hongo fresco fueron almacenadas a $4^{\circ} \mathrm{C}$ con tiempos de control de 0, 3, 6, 9, 12 y 15 días, utilizando tres tipos de empaque y tres atmósferas modificadas, con un peso aproximado de $150 \mathrm{~g} /$ empaque. Se empleó una empacadora al vació (Komet SD 320, Talsa S.A, Colombia) para realizar el sellado de la película a la bandeja, y un mezclador de gases en acero inoxidable (Pbi Dansensor Map Mix 9000, USA). Las condiciones ambientales para las muestras envasadas a presión atmosférica fueron $\mathrm{T} \cong 20^{\circ} \mathrm{C}$ y $\mathrm{HR} \cong 65 \%$. Se evaluó la estabilidad de las muestras respecto a los parámetros fisicoquímicos. Las características de las 
atmósferas 1, 2 y 3 fueron ambiente (presión atmosférica), $100 \% \mathrm{~N}_{2}$ y mezcla $\left(10 \% \mathrm{O}_{2}\right.$, $10 \% \mathrm{CO}_{2}, 80 \% \mathrm{~N}_{2}$ ) respectivamente. Para los empaques:

Empaque 1. Bandeja de espuma de poliestireno (icopor) con dimensiones $12 \times 20 \times 1.5 \mathrm{~cm}$, recubiertas con película trasparente de cloruro de polivinilo (PVC), permeabilidad al vapor de agua: $0 \mathrm{~g} / \mathrm{m}^{2} / 24 \mathrm{~h} /$ atm, $T=25^{\circ} \mathrm{C} ; \quad \mathrm{O}_{2}: 620 \mathrm{~cm}^{3} / \mathrm{m}^{2} / 24 \mathrm{~h} / \mathrm{atm}$, $\mathrm{CO}_{2}: 4263 \mathrm{~cm}^{3} / \mathrm{m}^{2} / 24 \mathrm{~h} /$ atm (Alico S.A). Los hongos frescos almacenados en el empaque 1 y atmósfera 1 (envasado comercial) se utilizaron como muestra control.

Empaque 2. Bandeja de polietilentereftalato/ polietileno de baja densidad (PET/LDPE, permeabilidad despreciable), con dimensiones $13 \times 18 \times 4.5 \mathrm{~cm}$, recubierta con laminado de polipropileno biorientado/polietileno de baja densidad (BOPP/LDPE) con $54 \mu \mathrm{m}$ de espesor, permeabilidad al vapor de agua: $0.3 \mathrm{~g} / \mathrm{m}^{2} / 24 \mathrm{~h} /$ atm, $\mathrm{T}=25^{\circ} \mathrm{C} ; \mathrm{O}_{2}: 3400 \mathrm{~cm}^{3} /$ $\mathrm{m}^{2} / 24 \mathrm{~h} /$ atm, $\mathrm{CO}_{2}: 12000 \mathrm{~cm}^{3} / \mathrm{m}^{2} / 24 \mathrm{~h} /$ atm (Alico S.A).

Empaque 3. Bandeja de polietilentereftalato/ polietileno de baja densidad (PET/LDPE, permeabilidad despreciable), recubierta con laminado polietileno de baja densidad (LDPE) con permeabilidad al vapor de agua: $23.2 \mathrm{~g} / \mathrm{m}^{2} / 24 \mathrm{~h} / \mathrm{atm}, \mathrm{T}=25^{\circ} \mathrm{C} ; \mathrm{O}_{2}$ : $4000 \mathrm{~cm}^{3} / \mathrm{m}^{2} / 24 \mathrm{~h} /$ atm, $\mathrm{CO}_{2} 8000 \mathrm{~cm}^{3} /$ $\mathrm{m}^{2} / 24 \mathrm{~h} / \mathrm{atm}$, y dimensiones $13 \times 18 \times 4.5$ $\mathrm{cm}$ (Alico S.A). Las combinaciones generan 7 tratamientos a evaluación: Atmósfera 1Empaque 1 o muestra control; Atmósfera 1- Empaque 2; Atmósfera 1- Empaque 3; Atmósfera 2- Empaque 2; Atmósfera 2- Empaque 3; Atmósfera 3- Empaque 2; Atmósfera 3- Empaque 3.
Caracterización del espacio de cabeza. Se determinó el contenido $\mathrm{O}_{2}$ y $\mathrm{CO}_{2}$ presentes en el espacio de cabeza de los empaques y atmósferas modificadas durante el tiempo de almacenamiento, utilizando un chequeador de gases en acero inoxidable (Pbi Dansensor Map Mix 9000, Dansensor, USA).

Análisis estadístico. Se utilizó la herramienta estadística STATGRAPHICS plus versión 5.1 , a partir de un modelo multifactorial completamente aleatorizado de tres factores de efectos fijos: empaque, atmósfera de envasado y tiempo de almacenamiento. Para cada tipo de atmósfera se realizó un análisis independiente a partir de ANOVAS, utilizando el método LSD (mínimas diferencias significativas) como método de comparaciones múltiples, con un nivel de confianza del 95\%.

\section{RESULTADOS}

Evolución de los parámetros fisicoquímicos. Las tablas 1, 2 y 3 presentan los valores medios y las desviaciones estándar de los parámetros fisicoquímicos para las muestras almacenadas en los empaques 1 , 2 y 3 respectivamente, envasadas en las atmósferas 1,2 y 3 .

Para todos los empaques, se encontraron diferencias significativas $(p<0.05)$ por efecto del tiempo de almacenamiento y la atmosfera de envasado en los parámetros ${ }^{\circ}$ Brix y $\mathrm{pH}$, mientras que para la $\mathrm{H}$ se presentaron diferencias por efecto del tipo de atmósfera de envasado para los empaques 2 y 3 .

Tabla 1. Características fisicoquímicas del hongo fresco en empaque 1 - atmósfera 1.

\begin{tabular}{ccccccc}
\hline & 0 días & 3 días & 6 días & 9 días & 12 días & 15 días \\
\hline $\mathrm{H}(\%)$ & $90.3 \pm 0.9$ & $89.2 \pm 1.5$ & $89.3 \pm 1.4$ & $90.5 \pm 1.1$ & $91.3 \pm 0.8$ & $91.8 \pm 0.7$ \\
$\mathrm{a}_{\mathrm{w}}$ & $0.994 \pm 0.002$ & $0.993 \pm 0.001$ & $0.994 \pm 0.001$ & $0.994 \pm 0.001$ & $0.994 \pm 0.001$ & $0.994 \pm 0.001$ \\
$\mathrm{pH}$ & $6.47 \pm 0.03$ & $6.44 \pm 0.01$ & $6.17 \pm 0.02$ & $6.13 \pm 0.02$ & $6.43 \pm 0.03$ & $6.19 \pm 0.05$ \\
Acidez (\%) & $0.20 \pm 0.01$ & $0.21 \pm 0.01$ & $0.19 \pm 0.01$ & $0.2 \pm 0.01$ & $0.2 \pm 0.01$ & $0.2 \pm 0.01$ \\
${ }^{\circ}$ Brix & $4.3 \pm 0.1$ & $4.7 \pm 0.1$ & $7.0 \pm 0.2$ & $6.4 \pm 0.1$ & $5.0 \pm 0.1$ & $4.7 \pm 0.1$ \\
Cloruros(\%) & $1.8 \pm 0.1$ & $2.0 \pm 0.1$ & $1.7 \pm 0.2$ & $2.1 \pm 0.1$ & $1.9 \pm 0.1$ & $1.8 \pm 0.2$ \\
\hline
\end{tabular}


Tabla 2. Características fisicoquímicas del hongo fresco en empaque 2.

\begin{tabular}{|c|c|c|c|c|c|c|}
\hline \multicolumn{7}{|c|}{ Atmósfera 1} \\
\hline & o días & 3 días & 6 días & 9 días & 12 días & 15 días \\
\hline $\mathrm{H}(\%)$ & $94.3 \pm 0.5$ & $90.1 \pm 1.3$ & $91.6 \pm 0.7$ & $94.8 \pm 0.6$ & $91.7 \pm 0.6$ & $94.6 \pm 0.4$ \\
\hline$a_{w}$ & $0.994 \pm 0.001$ & $0.996 \pm 0.001$ & $0.996 \pm 0.001$ & $0.997 \pm 0.001$ & $0.997 \pm 0.001$ & $0.997 \pm 0.001$ \\
\hline $\mathrm{pH}$ & $6.25 \pm 0.05$ & $6.17 \pm 0.05$ & $6.03 \pm 0.12$ & $5.91 \pm 0.05$ & $6.22 \pm 0.04$ & $6.00 \pm 0.08$ \\
\hline Acidez (\%) & $0.15 \pm 0.01$ & $0.17 \pm 0.01$ & $0.17 \pm 0.01$ & $0.16 \pm 0.01$ & $0.19 \pm 0.01$ & $0.19 \pm 0.01$ \\
\hline${ }^{\circ}$ Brix & $2.3 \pm 0.3$ & $3.0 \pm 0.1$ & $5.4 \pm 0.1$ & $4.6 \pm 0.3$ & $3.3 \pm 0.2$ & $2.9 \pm 0.1$ \\
\hline Cloruros(\%) & $2.0 \pm 0.2$ & $2.1 \pm 0.2$ & $2.3 \pm 0.3$ & $2.1 \pm 0.1$ & $2.1 \pm 0.1$ & $2.1 \pm 0.2$ \\
\hline \multicolumn{7}{|c|}{ Atmósfera 2} \\
\hline & O días & 3 días & 6 días & 9 días & 12 días & 15 días \\
\hline H (\%) & $94.3 \pm 0.5$ & $89.6 \pm 1.7$ & $91.9 \pm 0.2$ & $94.5 \pm 0.6$ & $91.5 \pm 0.8$ & $92.3 \pm 0.7$ \\
\hline$a_{w}$ & $0.994 \pm 0.001$ & $0.996 \pm 0.001$ & $0.996 \pm 0.001$ & $0.996 \pm 0.001$ & $0.997 \pm 0.001$ & $0.996 \pm 0.001$ \\
\hline $\mathrm{pH}$ & $6.25 \pm 0.05$ & $6.08 \pm 0.02$ & $5.75 \pm 0.07$ & $5.80 \pm 0.07$ & $6.15 \pm 0.03$ & $5.98 \pm 0.08$ \\
\hline Acidez (\%) & $0.15 \pm 0.01$ & $0.19 \pm 0.02$ & $0.18 \pm 0.01$ & $0.19 \pm 0.01$ & $0.19 \pm 0.01$ & $0.20 \pm 0.03$ \\
\hline${ }^{\circ}$ Brix & $3.1 \pm 0.1$ & $3.7 \pm 0.1$ & $6.0 \pm 0.2$ & $5.2 \pm 0.1$ & $3.7 \pm 0.1$ & $3.6 \pm 0.1$ \\
\hline Cloruros(\%) & $2.0 \pm 0.2$ & $2.0 \pm 0.1$ & $2.0 \pm 0.1$ & $2.0 \pm 0.1$ & $2.1 \pm 0.2$ & $2.1 \pm 0.2$ \\
\hline \multicolumn{7}{|c|}{ Atmósfera 3} \\
\hline & o días & 3 días & 6 días & 9 días & 12 días & 15 días \\
\hline $\mathrm{H}(\%)$ & $94.3 \pm 0.5$ & $89.4 \pm 1.7$ & $92.2 \pm 0.4$ & $93.9 \pm 1.2$ & $92.3 \pm 0.6$ & $93.2 \pm 0.5$ \\
\hline$a_{w}$ & $0.994 \pm 0.001$ & $0.996 \pm 0.001$ & $0.996 \pm 0.001$ & $0.997 \pm 0.001$ & $0.996 \pm 0.001$ & $0.997 \pm 0.001$ \\
\hline $\mathrm{pH}$ & $6.25 \pm 0.05$ & $6.21 \pm 0.04$ & $6.30 \pm 0.02$ & $6.11 \pm 0.02$ & $6.38 \pm 0.05$ & $5.96 \pm 0.08$ \\
\hline Acidez (\%) & $0.15 \pm 0.01$ & $0.19 \pm 0.01$ & $0.16 \pm 0.01$ & $0.16 \pm 0.01$ & $0.17 \pm 0.02$ & $0.12 \pm 0.01$ \\
\hline${ }^{\circ}$ Brix & $3.1 \pm 0.1$ & $3.4 \pm 0.1$ & $5.2 \pm 0.1$ & $4.1 \pm 0.2$ & $3.4 \pm 0.1$ & $3.0 \pm 0.1$ \\
\hline Cloruros(\%) & $2.0 \pm 0.2$ & $2.1 \pm 0.1$ & $2.1 \pm 0.1$ & $2.3 \pm 0.1$ & $2.1 \pm 0.1$ & $2.0 \pm 0.1$ \\
\hline
\end{tabular}

Tabla 3. Características fisicoquímicas del hongo fresco en empaque 3

Atmósfera 1

\begin{tabular}{|c|c|c|c|c|c|c|}
\hline & 0 días & 3 días & 6 días & 9 días & 12 días & 15 días \\
\hline $\mathrm{H}(\%)$ & $92.4 \pm 0.5$ & $89 \pm 1.1$ & $91.2 \pm 0.6$ & $92.3 \pm 0.9$ & $89 \pm 0.7$ & $90.8 \pm 0.5$ \\
\hline$a_{w}$ & $0.995 \pm 0.001$ & $0.995 \pm 0.001$ & $0.995 \pm 0.001$ & $0.996 \pm 0.001$ & $0.996 \pm 0.001$ & $0.996 \pm 0.001$ \\
\hline $\mathrm{pH}$ & $6.33 \pm 0.06$ & $6.27 \pm 0.06$ & $6.36 \pm 0.05$ & $6.26 \pm 0.07$ & $6.15 \pm 0.07$ & $6.44 \pm 0.08$ \\
\hline Acidez (\%) & $0.24 \pm 0.02$ & $0.17 \pm 0.01$ & $0.18 \pm 0.02$ & $0.25 \pm 0.01$ & $0.21 \pm 0.02$ & $0.21 \pm 0.01$ \\
\hline${ }^{\circ}$ Brix & $3.9 \pm 0.3$ & $3.8 \pm 0.3$ & $4.7 \pm 0.4$ & $4.2 \pm 0.3$ & $3.9 \pm 0.1$ & $3.8 \pm 0.3$ \\
\hline Cloruros(\%) & $2.2 \pm 0.1$ & $2.3 \pm 0.2$ & $2.3 \pm 0.3$ & $2.8 \pm 0.2$ & $2.4 \pm 0.2$ & $2.6 \pm 0.3$ \\
\hline \multicolumn{7}{|c|}{ Atmósfera 2} \\
\hline & 0 días & 3 días & 6 días & 9 días & 12 días & 15 días \\
\hline $\mathrm{H}(\%)$ & $92.4 \pm 0.5$ & $89.8 \pm 1.1$ & $90.1 \pm 0.3$ & $90.7 \pm 0.4$ & $90.7 \pm 0.5$ & $92 \pm 0.7$ \\
\hline$a_{w}$ & $0.995 \pm 0.001$ & $0.997 \pm 0.001$ & $0.996 \pm 0.001$ & $0.996 \pm 0.001$ & $0.997 \pm 0.001$ & $0.996 \pm 0.001$ \\
\hline $\mathrm{pH}$ & $6.33 \pm 0.06$ & $6.21 \pm 0.07$ & $6.33 \pm 0.11$ & $6.11 \pm 0.04$ & $6.10 \pm 0.04$ & $6.22 \pm 0.04$ \\
\hline Acidez (\%) & $0.24 \pm 0.02$ & $0.15 \pm 0.02$ & $0.18 \pm 0.01$ & $0.24 \pm 0.01$ & $0.20 \pm 0.01$ & $0.19 \pm 0.01$ \\
\hline${ }^{\circ}$ Brix & $3.9 \pm 0.3$ & $3.4 \pm 0.3$ & $3.8 \pm 0.2$ & $3.5 \pm 0.2$ & $3.6 \pm 0.2$ & $3.3 \pm 0.2$ \\
\hline Cloruros(\%) & $2.2 \pm 0.1$ & $2.1 \pm 0.2$ & $2.1 \pm 0.1$ & $2.5 \pm 0.2$ & $2.1 \pm 0.2$ & $2.1 \pm 0.1$ \\
\hline \multicolumn{7}{|c|}{ Atmósfera 3} \\
\hline & O días & 3 días & 6 días & 9 días & 12 días & 15 días \\
\hline $\mathrm{H}(\%)$ & $92.4 \pm 0.5$ & $89 \pm 0.8$ & $90.6 \pm 0.6$ & $93.9 \pm 1.2$ & $91.1 \pm 1.0$ & $93.0 \pm 0.5$ \\
\hline$a_{w}$ & $0.995 \pm 0.001$ & $0.997 \pm 0.001$ & $0.996 \pm 0.001$ & $0.996 \pm 0.001$ & $0.996 \pm 0.001$ & $0.996 \pm 0.001$ \\
\hline $\mathrm{pH}$ & $6.33 \pm 0.06$ & $6.53 \pm 0.15$ & $6.21 \pm 0.07$ & $6.18 \pm 0.08$ & $6.16 \pm 0.08$ & $6.45 \pm 0.06$ \\
\hline Acidez (\%) & $0.24 \pm 0.02$ & $0.20 \pm 0.00$ & $0.17 \pm 0.02$ & $0.23 \pm 0.03$ & $0.22 \pm 0.01$ & $0.16 \pm 0.01$ \\
\hline${ }^{\circ}$ Brix & $3.9 \pm 0.3$ & $3.8 \pm 0.2$ & $3.0 \pm 0.7$ & $3.3 \pm 0.3$ & $4.5 \pm 0.4$ & $3.1 \pm 0.2$ \\
\hline Cloruros(\%) & $2.2 \pm 0.1$ & $2.1 \pm 0.1$ & $2.1 \pm 0.2$ & $2.7 \pm 0.2$ & $2.4 \pm 0.1$ & $2.0 \pm 0.2$ \\
\hline
\end{tabular}


Evolución del color. Las figuras 1 y 2 presentan los valores medios para los parámetros $L^{*}, a^{*}$ y $b^{*}$ en hongos comestibles Pleurotus ostreatus en la muestra control y el empaque 2 y 3 (atmósferas 1, 2 y 3 ).

Para la muestra control, los factores tiempo y cara fueron significativos $(p<0.05)$ en todos los parámetros, siendo las muestras $\mathrm{CR}<\mathrm{CL}$ para $\mathrm{L}^{*}$ y las $\mathrm{CR}>\mathrm{CL}$ para a*y b* Para los hongos envasados en empaque 2, los factores tiempo y cara, fueron significativos para los parámetros $L^{*}$ y a* en todas las atmósferas, mientras que para $b^{*}$, solo tuvo efecto el tiempo. Para $L^{*}$, se observaron valores relativamente similares en las 3 atmósferas, dentro de un rango de variación entre 70-79, que lo ubica dentro de una luminosidad clara.

Para los hongos envasados en el empaque 3, los factores tiempo, atmósfera y cara fueron significativos $(p<0.05)$ para las variables $L^{*}, a^{*}$ y $b^{*}$, pero en rangos de variación bajos. Para todas las atmósferas, la tendencia de los valores de L*, a* y b* con el tiempo fue similar en ambas caras, siendo las muestras CL más clara y de menor saturación en la escala de los grises que las muestras CR

Evolución de la textura. La figura 3 presenta los valores medios de la fuerza máxima o fuerza de ruptura de los hongos envasados en las atmósferas 1,2 y 3 y empaques 1,2 y 3 durante el almacenamiento. EI ANOVA presentó diferencias significativas $(p<0.05)$ por efecto del factor tiempo y el tipo de empaque en todas las atmósferas.

Para la atmósfera ambiente, las muestras presentaron un comportamiento relativamente similar, manteniéndose en un rango de valores entre 1900 y $1300 \mathrm{gf}$. Para la atmósfera 2, existió una tendencia a disminuir la textura del producto en los empaques 2 y 3 , a los 6 y 9 días respectivamente. En la atmósfera 3, el comportamiento de los empaques 2 y 3 fue similar, con coeficientes de variabilidad amplios y diferencias solo al tercer día.
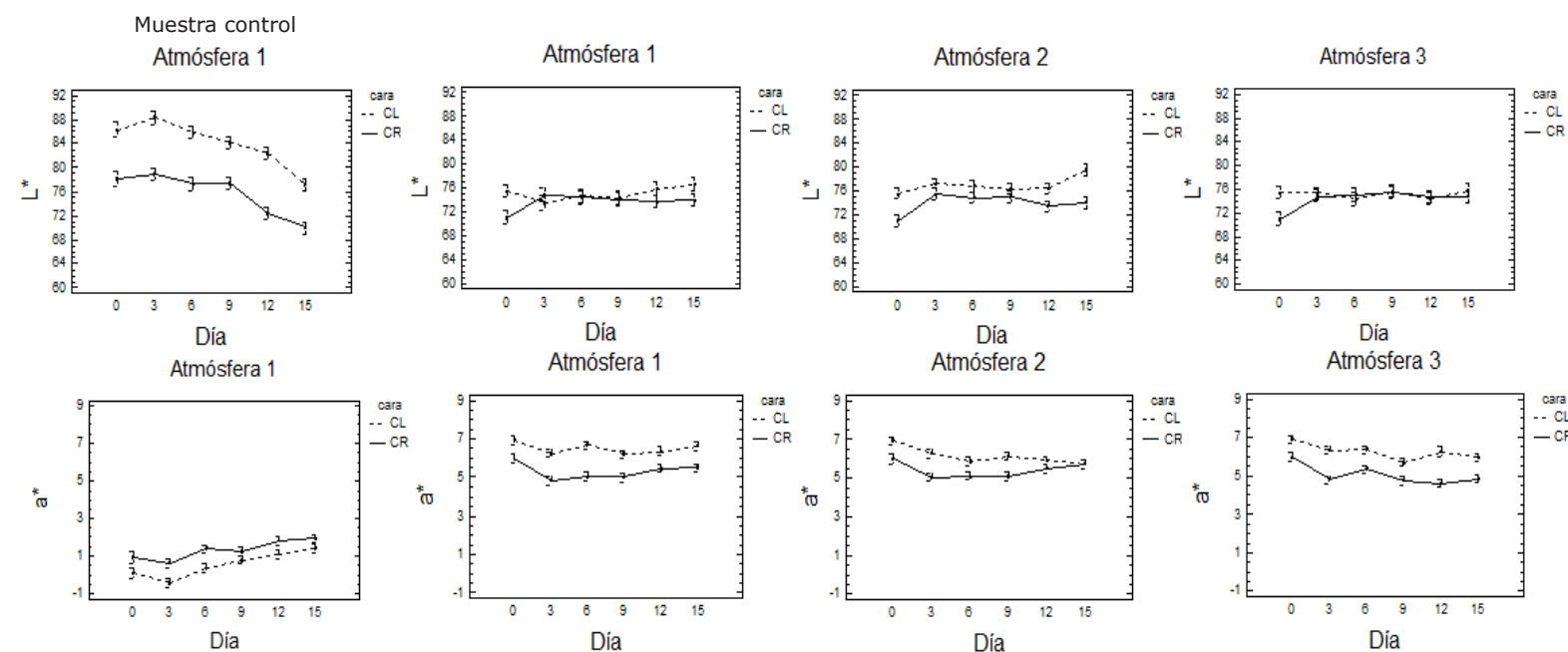

Atmósfera 1

Atmósfera 2
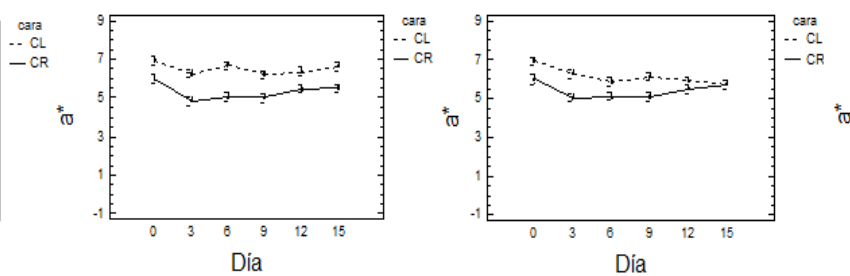

Atmósfera 3

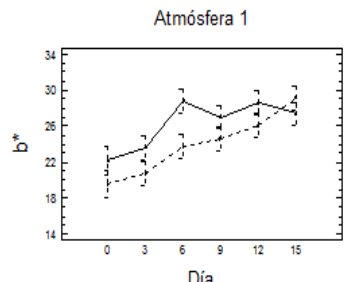

Día

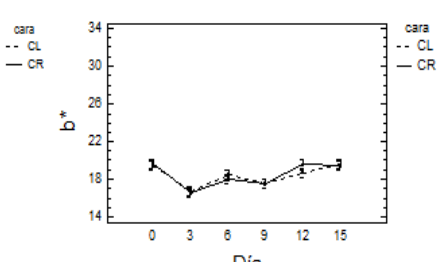

Día

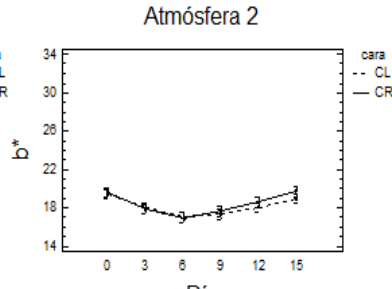

Día

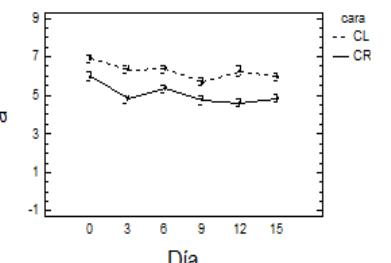

Atmósfera 3

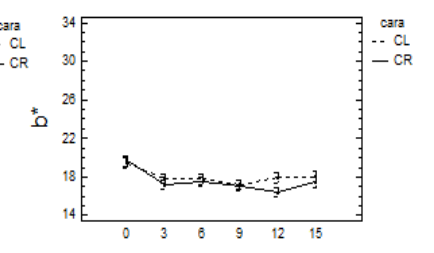

Día

Figura 1. Valores medios con los intervalos LSD (95\%) de L*, $a^{*}$ y b* en hongos comestibles (Pleurotus ostreatus) para las caras lisa (CL) y rugosa (CR) de hongos frescos empacados en la muestra control y el empaque 2 (atmósferas 1,2 y 3 ) durante el tiempo de almacenamiento. 
Atmósfera 1

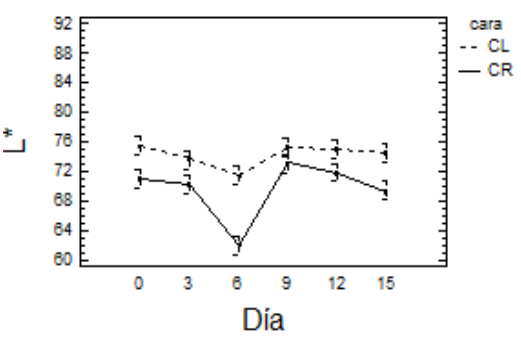

Atmósfera 1

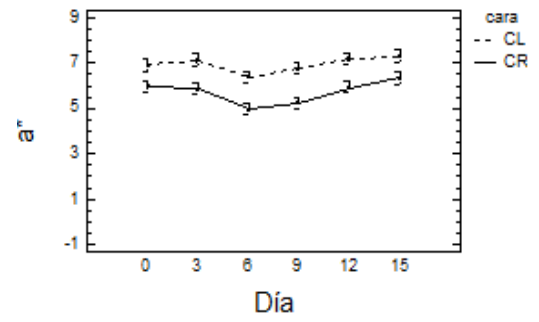

Atmósfera 1

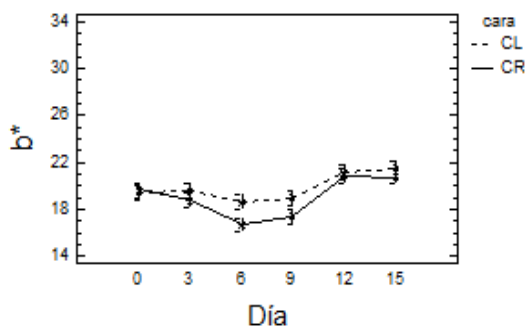

Atmósfera 2

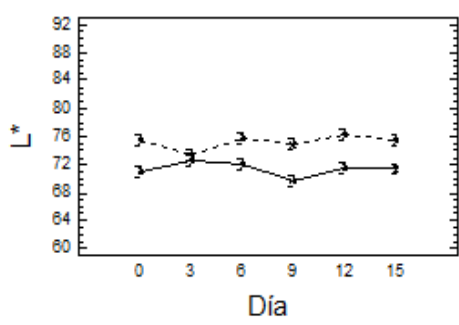

Atmósfera 2

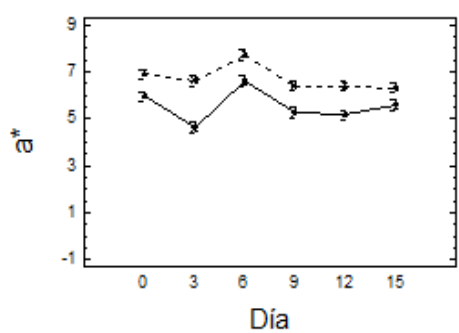

Atmósfera 2

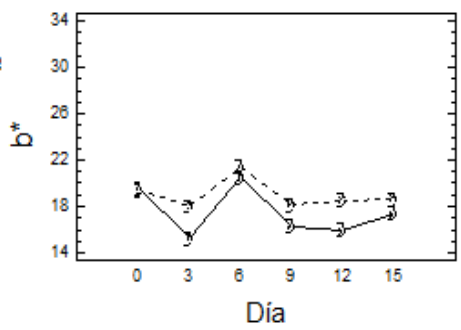

Atmósfera 3
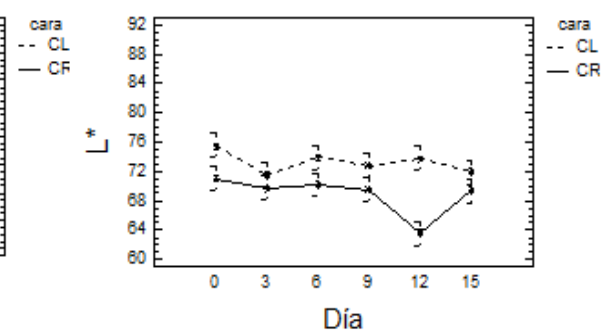

Atmósfera 3
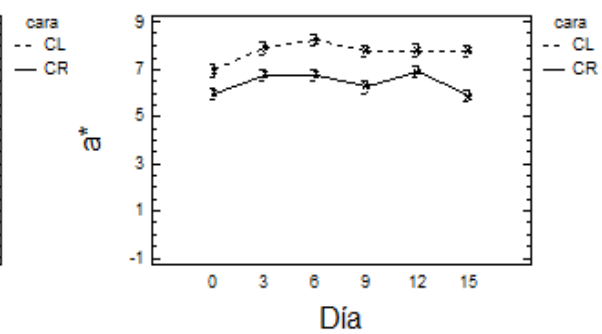

Atmósfera 3

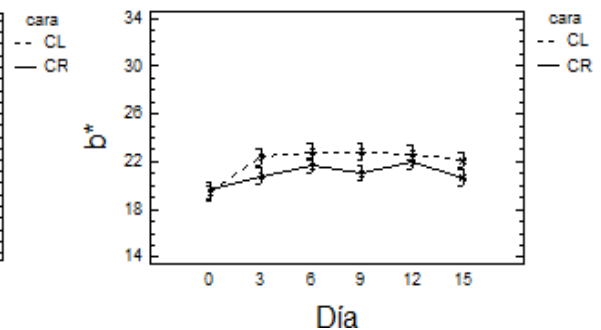

Figura 2. Valores medios con los intervalos LSD (95\%) de L*, a* y b* en hongos comestibles (Pleurotus ostreatus) para las caras lisa (CL) y rugosa (CR) de hongos frescos empacados en la muestra control y el empaque 3 (atmósferas 1,2 y 3 ) durante el tiempo de almacenamiento.
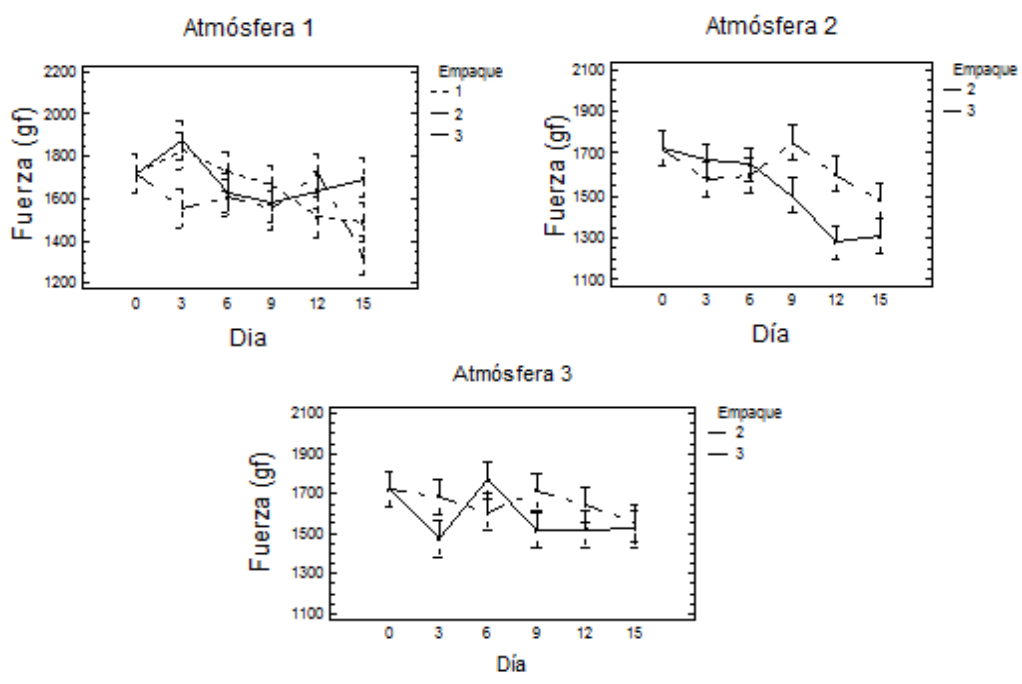

Figura 3. Valores medios con los intervalos LSD (95\%) del parámetro de textura fuerza de penetración, para hongos comestibles (Pleurotus ostreatus) envasados bajo todos los empaques y atmosferas mencionadas durante el almacenamiento. 
Cambios en la composición de los gases en el espacio de cabeza. La figura 4 presenta los cambios en la actividad respiratoria del hongo.

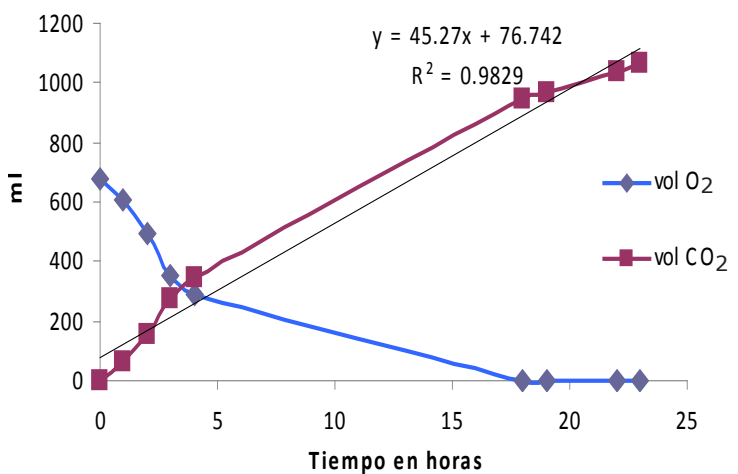

Figura 4. Curva de respiración del hongo comestible (Pleurotus ostreatus) a $4^{\circ} \mathrm{C}$, durante 24 horas.

La tabla 4 muestra los valores en el \% de $\mathrm{O}_{2}$ y $\mathrm{CO}_{2}$ para cada combinación de atmósfera y empaque. Para el empaque 2, el comportamiento del $\mathrm{O}_{2}$ y el $\mathrm{CO}_{2}$ en el espacio de cabeza, alcanzó una condición de anaerobiosis $\left(\approx 0 \% \mathrm{O}_{2}\right)$ antes del día 4 en las atmósferas 1 y 3 . La generación de $\mathrm{CO}_{2}$ alcanzó valores entre el 20 y $30 \%$ entre los días 4 y 12.

Para el empaque 3, se alcanzó la condición de anaerobiosis el día 9 en las atmósferas

Tabla 4. Valores de $\% \mathrm{O}_{2}$ y $\mathrm{CO}_{2}$ para empaque 2 y 3 , de acuerdo a la atmosfera de almacenamiento.

\begin{tabular}{ccccccc}
\hline \multicolumn{7}{c}{ Empaque 2 } \\
\hline Tiempo & \multicolumn{7}{c}{ Atmósfera 1 } & \multicolumn{2}{c}{ Atmósfera 2 } & \multicolumn{2}{c}{ Atmósfera 3 } \\
\hline & $\mathrm{O}_{2}$ & $\mathrm{CO}_{2}$ & $\mathrm{O}_{2}$ & $\mathrm{CO}_{2}$ & $\mathrm{O}_{2}$ & $\mathrm{CO}_{2}$ \\
0 días & 20.9 & 0 & 0 & 0 & 10.0 & 10.0 \\
4 días & 0 & 21.6 & 0 & 12.3 & 0 & 21.0 \\
9 días & 0.1 & 24.7 & 0 & 18.9 & 0 & 25.5 \\
1 12días & 0.2 & 30.9 & 0 & 21.0 & 0.2 & 29.7 \\
\hline & & & Empaque 3 & & \\
\hline Tiempo & Atmósfera 1 & Atmósfera 2 & Atmósfera 3 \\
\hline & $\mathrm{O}_{2}$ & $\mathrm{CO}_{2}$ & $\mathrm{O}_{2}$ & $\mathrm{CO}_{2}$ & $\mathrm{O}_{2}$ & $\mathrm{CO}_{2}$ \\
\hline 0 días & 20.9 & 0 & 0 & 0 & 10 & 10 \\
\hline 4 días & 9.6 & 5,1 & 0 & 7.4 & 4.2 & 4.7 \\
\hline 9 días & 0 & 7.1 & 0 & 10.8 & 0.9 & 6.0 \\
\hline 12días & 0.1 & 10.3 & 0 & 13.6 & 10.3 & 0.5 \\
\hline
\end{tabular}

1 y 3, siendo mayor los niveles de $\mathrm{O}_{2}$ que en el empaque 2. Los niveles de $\mathrm{CO}_{2}$ en la atmósfera 1 se incrementaron hasta un valor del $10 \%$, mientras que en la atmósfera 3 , inicia con el $10 \%$ del gas de envasado y presenta una reducción hasta valores de $0.5 \%$.

Para los empaques 2 y 3, la atmósfera 2 (atmósfera inerte), presentó como era esperado, niveles de $\mathrm{O}_{2}$ nulos, con una tasa de producción de $\mathrm{CO}_{2}$ hasta un $21 \%$ y $13.6 \%$, respectivamente.

\section{DISCUSIÓN}

Evolución de los parámetros fisicoquímicos. Los cambios en los ${ }^{\circ}$ Brix, durante el almacenamiento, están asociados principalmente al desdoblamiento de los carbohidratos presentes (rompimiento de los polisacáridos en monosacáridos) (25) y a la deshidratación progresiva del producto por la diferencia de potencial químico entre el producto y la atmósfera (26); cuyo resultado es el incremento del contenido de azucares en el producto durante los primeros seis días y posteriormente tiende a establecer el equilibrio al final del almacenamiento. Este comportamiento ocurrió en todos los empaques, con una mayor intensidad en el empacado comercial (muestra control), seguido del empaque 2 para la atmósfera 2, y concuerda con estudios previos realizados en Agrocybe chaxingu empacados en atmósfera modificada $\left(5 \% \mathrm{O}_{2}, 10 \% \mathrm{CO}_{2}\right.$, $85 \% \mathrm{~N}_{2}$ ) (17).

Para el hongo fresco $(\mathrm{t}=0)$ los parámetros de aw, humedad, $\mathrm{pH}^{\circ}{ }^{\circ}$ Brix fueron similares a los reportados en otras investigaciones (27-29), mientras que la acidez presentó valores superiores $(4.00 \pm 0.23 \%$ frente $1.95 \pm 0.01 \%)$.

En general, todas las muestras presentaron diferencias estadísticas significativas en los parámetros fisicoquímicos del hongo fresco por efecto de los factores tiempo de almacenamiento, empaque y tipo de atmósfera; los rangos de variación se consideraron aceptables y enmarcados en 
las características propias del producto, como ente biológico que continua con sus procesos metabólicos.

Evolución del color. Para la muestra control los datos obtenidos de L* mostraron valores en $\mathrm{CR}<\mathrm{CL}$ y para los parámetros $a * y$ b* valores en $C R>C L$. Estos últimos concuerdan con los reportados por Cortés et al (28) para los parámetros a* y b* en hongos de la misma variedad; sin embargo los valores de $L *$ fueron mayores para ambas caras en el presente estudio. Estas variaciones se atribuyen a diversos factores como el sustrato utilizado durante la siembra, las condiciones de cosecha y la composición final del producto. La evolución de los parámetros de color con el tiempo, refleja una cinética similar para ambas caras, presentando inicios de pardeamiento al final del almacenamiento, lo cual se identifica por la disminución de $L^{*}$ a valores inferiores a 70 y el incremento de la cromaticidad $a *$ y b*, pero permaneciendo en la cromaticidad de la escala de grises.

Los bajos rangos de variación de $L^{*}$, provocan diferencias difícilmente apreciables entre las caras del hongo y a través del tiempo de almacenamiento. Para a* y b*, el comportamiento fue similar para las atmósferas, en un rango de valores entre 4-7 (a*) y 16-20 (b*). Esta combinación de parámetros, produce un nivel de saturación de color bajo en escala de grises; así estas diferencias mínimas no son apreciables a simple vista y pueden no ser percibidas por el observador (como medidor subjetivo del color).

Las diferencias entre las caras del hongo a través del tiempo de almacenamiento son atribuidas más a la variabilidad propia del producto y la irregularidad de la superficie de la $C R$, que a la interacción de los gases de la atmósfera con el sustrato alimentario.

A pesar de los cambios detectados por el análisis estadístico en los parámetros de color, debido a los factores de tiempo, atmósfera y cara, los rangos de variación de $L^{*}, a^{*}$ y $b^{*}$ - no representan diferencias apreciables para el observador, lo que permite concluir que las diferencias de color por el uso del empaque en las 3 atmósferas no son relevantes. Investigaciones realizadas por Villaescusa y Gil (11), reportan que el parámetro b* es el más representativo para detectar las diferencias en el color del hongo, lo cual sustenta lo expuesto anteriormente.

En general, no se presentó pardeamiento enzimático ni diferencias de color apreciables a nivel del observador a través del tiempo en los empaques y atmósferas de estudio, contrario a lo reportado en otras variedades de hongos en atmósferas modificadas (14). Se pueden observar algunas ligeras fluctuaciones más por variaciones propias del producto.

En conclusión los cambios de color fueron más acentuados en la muestra control, alcanzando un ligero pardeamiento al final del almacenamiento. Para los empaques 2 y 3 , en las 3 atmósferas de envasado, se presentaron diferencias significativas por efecto de los factores de estudio con uniformidad en el rango estrecho de valores a través del tiempo de almacenamiento, sin llegar a ser realmente apreciables por el observador.

Evolución de la textura. Para la atmósfera ambiente el rango de valores de la fuerza de ruptura entre 1900 y 1300 gf., corresponde a una ruptura de valor medio para este tipo de material (28). Las variaciones se atribuyen a los daños generados por los cambios metabólicos y de senescencia del producto (29), además de la acción de algunas enzimas del grupo de las hidrolasas, presentes en frutas, bacterias, hongos y levaduras (30).

La tendencia a disminuir la textura del producto en la atmósfera 2 en combinación con los empaques 2 y 3 , puede asociarse a un debilitamiento de la estructura celular debido a procesos fermentativos y a la respiración facultativa del hongo (30). Villaescusa y Gil (11), detectaron procesos fermentativos en Pleurotus ostreatus, envasado en empaques de PVC y polietileno de baja densidad (LDPE). 
La textura presentó diferencias significativas por efecto de los factores tiempo y empaque en todas las atmósferas, con una disminución en la resistencia mecánica, debido a los posibles procesos fermentativos y al deterioro por la alta tasa metabólica.

\section{Cambios en la composición de los gases en el espacio de cabeza. LOS} valores encontrados en producción de $\mathrm{CO}_{2}$ se ajustan a los reportados por Kim et al (10), confirmando la alta tasa respiratoria del producto.

La condición de anaerobiosis $\left(\approx 0 \quad \% \mathrm{O}_{2}\right)$ antes del día 4 en las atmósferas 1 y 3 para el empaque 2, ocurre rápidamente al suplirse las demandas fisiológicas, provocando una alta tasa inicial de respiración, asociada a la tensión poscosecha (fuerza capilar y tasa de realización de procesos biológicos normales) debida al corte del pie (tallo) antes del almacenamiento. Resultados similares en hongos de la misma variedad fueron obtenidos por Villaescusa y Gil (11). El mayor descenso de $\mathrm{O}_{2}$ para la atmósfera 1 , es ocasionado por las condiciones iníciales de la atmosfera que contiene un $11 \%$ más del mismo. Las altas concentraciones de $\mathrm{CO}_{2}$ (valores entre el 20 y $30 \%$ ) acumuladas al interior del empaque son atribuidas al mismo fenómeno de respiración, lo cual es similar para la mayoría de los vegetales, por lo que la modificación del espacio de cabeza sucede rápidamente de acuerdo con las propiedades de permeabilidad al gas de la película (10).

Los mayores niveles de $\mathrm{O}_{2}$ para el empaque 3 (comparado con el empaque 2) son debidos a su mayor permeabilidad. La reducción de los valores de $\mathrm{CO}_{2}$ para la atmósfera 3 es atribuido a los procesos de perdida por transpiración y permeabilidad del empaque.
El aumento en la concentración de $\mathrm{CO}_{2}$ para la atmósfera 2 (atmósfera inerte) en ambos empaques es debido a la facultad fermentativa del hongo. En general, los hongos son aeróbicos, pero también tienen la capacidad de ser microaerobios y en condiciones de almacenamiento con bajos niveles de $\mathrm{O}_{2}$, esta puede resultar en concentraciones insuficiente para un metabolismo aeróbico, donde los tejidos pueden iniciar una respiración anaeróbica, en la que la glucosa se transforma en ácido láctico o acetaldehído y etanol (fermentación). La concentración de $\mathrm{O}_{2}$ a la que se inicia la fermentación varía con los tejidos y se conoce como punto de extinción, el cual depende de numerosos factores como la especie, el cultivar, el grado de madurez y la temperatura pre y poscosecha (30).

La estructura del hongo, presentó una alta tasa de respiración en las atmósferas 1 y 3. La generación de $\mathrm{CO}_{2}$ en el Pleurotus ostreatus, debida a procesos respiratorios alcanzó al final del almacenamiento niveles máximos del orden del $30 \%$ en el empaque 2; mientras que para el empaque 3 , presentó una reducción desde el $10 \%$ hasta $0.5 \%$, asociado al balance entre el proceso de transpiración y la permeabilidad de la película. La atmósfera 2, presentó una tasa máxima de producción de $\mathrm{CO}_{2}$ en el empaque 2 , alcanzando niveles del $21 \%$.

En conclusión, los resultados obtenidos permiten identificar que los empaques y las atmósferas modificadas evaluadas, no influyen significativamente en las características fisicoquímicas y fisiológicas tratadas en esta investigación para el hongo Pleurotus ostreatus, lo que hace que el control (empaque comercial) sea más práctico por efecto de costos de producción. 


\section{REFERENCIAS}

1. Mayela BJ, Alanis MG, González de ME, García CL, Martínez F, Barbosa E. Calidad proteínica de tres cepas mejicanas de setas (Pleorotus ostreatus). ALAN 1999; 49 (1): 81-85.

2. Hincapié JG. Fertilización mineral del hongo comestible Pleurotus ostreatus [trabajo para optar al título de ingeniero agrónomo]. Medellín, Colombia: Universidad Nacional de Colombia., 1993.

3. Wang D, Sakoda A, Suzuki M. Biological efficiency and nutricional value of Pleorotus ostreatus cultivated on spent beer grain Bioresource Technol 2001; 78: 293-300.

4. Manzi P, Aguzi A, Pizzoferrato L. Nutritional value of mushroom widely consumed in Italy. Food Chem 2001; 73: 321-325.

5. Moradali M, Mostafavi $H$, Ghods S, Hedjaroude A. Immunomodulating and anticancer agents in the realm of macromycetes fungi (macrofungi). Int Immunopharmacol 2007; 7:701-724.

6. Cardona LF. Propiedades medicinales de algunos hongos asociados a la madera. Cron For Medio Ambient 2003; 18: 47-64.

7. Manzi $P$, Pizzoferrato L. Beta glucans in edible mushrooms. Food Chem 2000; 68: 315-318.

8. Acevedo ME, Gómez J, Martínez A, Medina J, Morneo Z, Pizarro J. Grupo Sim: Setas y Hongos, Corporación Colombiana Internacional. Inteligencia de mercados 2004; (21): 1-6.

9. Jacxsens L, Devlieghere F, Van der Steen C, Debevere J. Effect of high oxygen modified atmosphere packaging on microbial growth and sensorial qualities of fresh-cut produce. Int J Food Microbiol 2001; 71: 197-210.
10. Kim KM, Kob JA, Leec JS, Parkb HJ, Miford A. Effect of modified atmosphere packaging on the shelf-life of coated, whole and sliced mushrooms. LWT 2006; 39: 364-371.

11. Villaescusa R, Gil MI. Quality improvement of Pleurotus mushrooms by modified atmosphere packaging and moisture absorbers. Postharv Bio Technol 2003; 28: 169-179.

12. Fonseca SC, Oliveira FA, Brecht JK. Modelling respiration rate of fresh fruits and vegetables for modified atmosphere packages: a review. J Food Eng 2002; 52: 99-119.

13. Cliffe-Byrnes V, ÓBeirne D. Effects of washing treatment on microbial and sensory quality of modified atmosphere (MA) packaged fresh sliced mushroom (Agaricus bisporus). Postharv Bio Technol 2008; 48: 283-294.

14. Tano K, Oulé M, Doyon G, Lencki R, Arul J. Comparative evaluation of the effect of storage temperature fluctuation on modified atmosphere packages of selected fruit and vegetables. Postharv Bio Technol 2007; 46: 212-221.

15. Tao F, Zhang M, Yu H. Effect of vacuum cooling on physiological changes in the antioxidant system of mushroom under different storage conditions. J Food Eng 2007; 79: 1302-1309.

16. Tao, F., Zhang, M., Yu, H, Jincai, $S$. Effects of different storage conditions on chemical and physical properties of white mushrooms after vacuum cooling. J Food Eng 2006; 77: 545-549.

17. Li T, Zhang M, Wang S. Effects of modified atmosphere packaging with a silicon gum film as a window for gas exchange on Agrocybe chaxingu storage. Postharv Biol Technol 2007; 43: 343-350. 
18. Ares G, Parentelli C, Gambaro A, Lareo C, Lema P. Sensory shelf-life of shiitake mushrooms stored under passive modified atmosphere. Postharv Biol Technol 2006; 41: 191-197.

19. Lopez-Briones, G., Varoquaux, $\mathrm{P}_{\text {., }}$ Chambroy, Y., Bouquant, J., Bureau, G., \& Pascat, B. Storage of common mushroom under controlled atmospheres. Int J Food Sci Tech 1992; 27: 493-505.

20. Popa M., Stanescu M., Ilie A., Dumitrescu R., Vraci I. Some aspects regarding modified atmosphere packaging of mushrooms. En: Hägg M., Ahvenainen R., Evers AM., Tiilikkala, K. Quality Management of Fruits and Vegetables. Cambridge; 1999.

21. Henze, J. Storage and transport of Pleurotus mushrooms in atmospheres with high $\mathrm{CO} 2$ concentrations. Acta Hort 1989; 258: 579-584.

22. Instituto Colombiano de Normas Técnicas ICONTEC, Normas Técnicas Colombianas. Cuarta revisión. Bogotá; 1999.

23. Instituto Colombiano de Normas Técnicas ICONTEC, Normas Técnicas Colombianas. Cuarta revisión. Bogotá; 2000

24. AOAC. Official Methods of Analysis. 17th Edition. Galthersburg MD, USA: Association of Official Analytical Chemists; 2000.
25. Badui Jergal S. Química de los alimentos. Cuarta edición. México. 2006

26. Fito $P$, Andrés $A$, Barát $J$, Abors $A$. Introducción al secado de alimentos por aire caliente. España: Editorial Universidad Politécnica de Valencia; 2001.

27. García, A. Desarrollo de hongos comestibles, Pleurotus ostreatus mínimamente procesados fortificados con calcio, selenio y vitamina C por aplicación de la técnica de impregnación a vació [trabajo para optar al título de ingeniero de alimentos]. Bogotá, Colombia: Universidad Jorge Tadeo Lozano., 2007.

28. Cortés M, García A, Suárez H. Fortificación de hongos comestibles (Pleurotus ostreatus) con calcio, selenio y vitamina $\mathrm{C}$. Vitae 2007; 14(1):16-24.

29. Gras M, Vidal-Brotons D, Betoret N, Chiralt $A$, Fito $P$. The response of some vegetables to vacuum impregnation. Innov Food Sci Emerg 2002; 3:263-269.

30. Salisbury F, Ross C. Fisiología de las plantas. Madrid, España: Thomson International; 2000. 